\title{
Percepções do ambiente construído e sua associação com a caminhabilidade objetiva
}

\author{
Karina Guimarães Silva ${ }^{a} \mathbb{C}^{D}$, Ana Luiza Favarão Leão ${ }^{b}{ }^{(\mathbb{B}}$, \\ Mariana Ragassi Urbano $^{c}$ (i) e Milena Kanashiro ${ }^{d}$ \\ ${ }^{a}$ Universidade Estadual de Londrina, Departamento de Arquitetura e Urbanismo, Londrina, PR, Brasil. E- \\ mail: karina.guimaraes@uel.br \\ ${ }^{\mathrm{b}}$ Universidade Estadual de Londrina, Departamento de Arquitetura e Urbanismo, Programa de Pós- \\ Graduação em Arquitetura e Urbanismo, Londrina, PR, Brasil. E-mail: analuiza.favarao@uel.br \\ ${ }^{c}$ Universidade Estadual de Londrina, Departamento de Estatística, Programa de Pós-Graduação em \\ Arquitetura e Urbanismo, Londrina, PR, Brasil. E-mail: mrurbano@uel.br \\ ${ }^{d}$ Universidade Estadual de Londrina, Departamento de Arquitetura e Urbanismo, Programa de Pós- \\ Graduação em Arquitetura e Urbanismo, Londrina, PR, Brasil. E-mail: milena@uel.br
}

Submetido em 07 de julho de 2019. Aceito em 03 de novembro de 2019.

\begin{abstract}
Resumo. Com o crescente ônus do transporte motorizado, qualidades urbanas têm guiado pesquisas para entender a influência da forma urbana sobre o deslocamento ativo. Uma das estratégias para avaliar o ambiente é a caminhabilidade, entendida como a extensão em que características da forma urbana podem ou não ser condutivos ao caminhar. Esta qualidade pode ser analisada de forma subjetiva ou objetiva, considerando percepções ou quantificações de características do ambiente. Desta forma, o objetivo geral da pesquisa é analisar percepções do ambiente relacionadas a caminhabilidade em uma cidade média brasileira. Percepções foram extraídas e categorizadas da pesquisa de Origem-Destino realizada para o plano de mobilidade de um estudo de caso - Rolândia/PR. Um Índice foi sistematizado, como uma medida objetiva e composta da caminhabilidade, por setor censitário e analisado na sobreposição de mapas em relação às percepções pelo procedimento estatístico de Kruskall-Wallis. Resultados indicam que percepções de funcionalidade, estética e destinos demostraram maior relação com a caminhabilidade, enquanto percepções de segurança se mostraram menos relevantes. $O$ estudo contribui para diretrizes de mobilidade na incorporação de percepções ambientais como suporte para o deslocamento ativo para fomentar cidades sustentáveis.
\end{abstract}

Palavras-chave. ambiente construído, caminhabilidade, sustentabilidade, percepção.

\section{Introdução}

A relação direta entre o crescente nível de doenças crônicas e o sedentarismo da população mundial (Humpel et al., 2004; Bauman et al., 2012; Dewulf et al., 2012; Jáuregui et al., 2016) originou estudos de mobilidade ativa e planejamento urbano que objetivam a melhora na qualidade de vida urbana. Tal abordagem é contrária ao processo de crescimento das cidades na priorização do transporte motorizado em detrimento ao deslocamento ativo - a pé e de bicicleta.

Assim a caminhada passa a ser entendida como um componente importante da atividade física, acessível a todas as classes sociais e como comportamento ativo mais passível de influência pelo ambiente urbano (Leslie et al., 2005). Deste modo, a caminhabilidade é a qualidade espacial base para uma cidade sustentável (Southworth, 2005), definida como a extensão em que as 
características da forma urbana e o uso do solo podem ou não proporcionar segurança, acessibilidade e conectividade de acessos às rotas de pedestres (Shamsuddin, Hassan e Bilyamin, 2018)

As primeiras discussões relacionadas à influência do ambiente construído sobre o deslocamento ativo partem da discussão proposta por Cervero e Kockelman (1997), em que as características da forma urbana induzem o comportamento e as escolhas dos pedestres e definem três dimensões:

Densidade - bairros compactos, com maior proximidade entre origens e destinos, Diversidade - variedade de usos do solo e Design - aspectos qualitativos do bairro, características do projeto urbano.

Neste sentido, a qualidade da rota entre origens e destino (OD) exerce grandes influências sobre comportamentos ativos (Southworth, 2005), e observa-se nessas dimensões, atributos objetivos e subjetivos para avaliar a caminhabilidade (Lee et al., 2017). A metodologia de mensuração objetiva mais disseminada foi a proposta por (Frank et al., 2010) na sistematização de um índice de caminhabilidade. Este índice parte da combinação de fatores do ambiente construído: densidade residencial, densidade de intersecções, taxa de ocupação de lotes comerciais e entropia ou uso misto do solo (Frank et al., 2010). Evidências sólidas indicam a relação entre os atributos objetivos e a atividade de pedestres, ou seja, bairros que possuem taxas elevadas destes fatores ambientais possuem altos níveis de caminhabilidade (Frank et al., 2010).

Por outro lado, entende-se que apenas a forma da cidade não é suficiente para incentivar o deslocamento ativo.

Características ambientais devem estar correlacionados aos fatores cognitivos, ou seja, a percepção destas qualidades pelos moradores do bairro (Jáuregui et al., 2016). Assim, estudos qualitativos propõe a exploração das influências da percepção do ambiente sobre a atividade física, analisando sua relação com fatores psicológicos, culturais, variáveis individuais e níveis de satisfação de bairro (Kirtland et al., 2003; Pikora et al., 2003; Humpel et al., 2004; Leslie et al., 2005; Leslie e Cerin, 2008; Kamphuis et al., 2010; Mackenbach et al., 2016; Lee et al., 2017).

A partir da forma como atributos do ambiente são apreendidos pelo usuário, interferências diretas ocorrem na percepção (Baldock et al., 2019), Pikora et al. (2003) sistematiza variáveis ambientais relacionadas com o deslocamento ativo e define um framework (Figura 1). Os elementos foram categorizados em quatro dimensões que influenciam a percepção: Funcionalidade atributos físicos das calçadas e largura das ruas que influenciam nos aspectos estruturais fundamentais do espaço urbano, como o tipo de caminho, velocidade da via e tipos de intersecções, Segurança- elementos que proporcionam a segurança pessoal e de tráfego do ambiente, como maior iluminação do espaço e apoios para travessia de ruas Estética - atratividade da paisagem urbana e das vistas diversas através de manutenção das ruas, presença de jardins, dimensões das árvores e elementos arquitetônicos $e$ Destinos- facilidades comunitárias e comerciais do bairro, como escolas, parques, shoppings e pontos de ônibus.

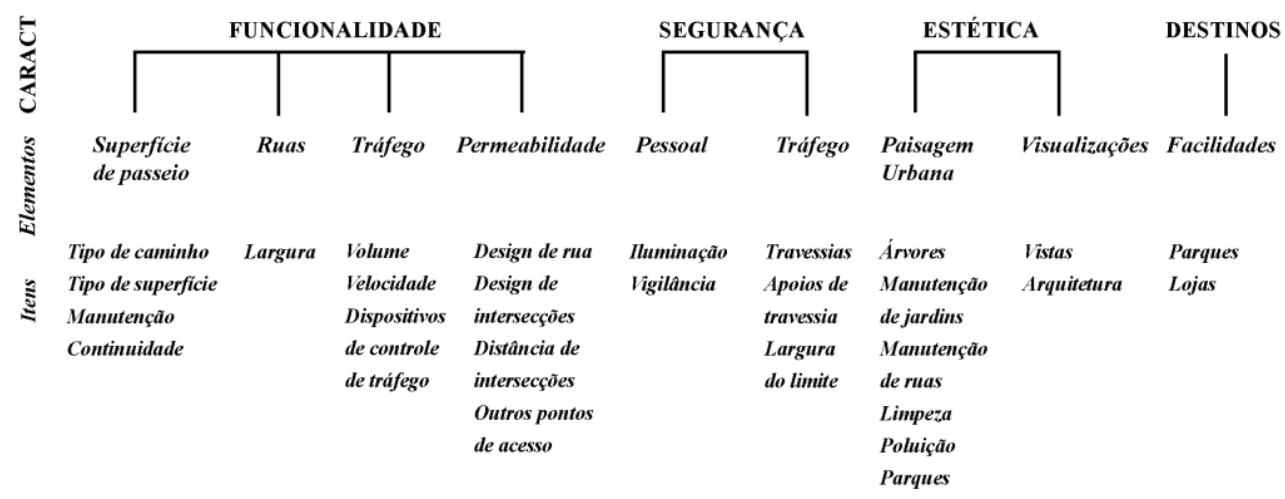

Figura 1. Framework de percepções ambientais (Fonte: Pikora et al., 2003, p.1698, traduzido pelas autoras, 2019). 
A combinação de atributos objetivos e subjetivos determina a satisfação com o bairro, por meio de fatores sociais e psicológicos (Lee et al., 2017) e, geralmente o nível de satisfação com o ambiente de caminhada estabelece-se via experiências físicas e visuais (Pikora et al., 2003). Assim, a satisfação com o bairro pode ser influenciada por dois domínios: o individual e aquele relacionado às características do design urbano (Basolo e Strong., 2002). Entretanto, é possível obter interações inversas entre os dois domínios: características ambientais entendidas como relacionadas com a caminhabilidade podem coexistir com baixos níveis de satisfação (Lee et al., 2017).

Em síntese, a análise de caminhabilidade, pode ser mensurada por meio de medições objetivas de características do ambiente ou de medições de percepção dos usuários. Os dados das medidas percebidas podem ser adquiridos por meio de questionários e, no caso de medidas objetivas, analisadas através de sistemas de informações geográficas (SIG) (Jáuregui et al., 2016; Kirtland et al., 2003). A abordagem combinada entre medidas objetivas e subjetivas mostraram associações em algumas pesquisas (Kirtland et al., 2003; Humpel $\mathrm{N}$ et al., 2004; Leslie e Cerin, 2008; Jáuregui et al., 2016) e a não relação entre as mesmas (Lee et al., 2017; Baldock et al., 2019).

Acrescenta-se às discussões de mensuração da caminhabilidade nas cidades brasileiras, os dados da Associação Nacional de Transportes Públicos (ANTP, 2018). A participação do transporte não motorizado (bicicletas e a pé) eleva-se com a redução do tamanho do município, sendo que aqueles que possuem de 60 a 100 mil habitantes apresentam maiores percentuais de deslocamento a pé. Portanto, quanto menor o porte do município maior é o deslocamento por transporte não motorizado e menor o uso do transporte coletivo. Tais questões apontam para a necessidade de avaliações para subsidiar políticas de mobilidade ativa em cidades médias e pequenas e, consequentemente, o entendimento das associações entre mensurações objetivas e subjetivas da caminhabilidade.

Sabendo que a qualidade urbana da caminhabilidade exerce influências sobre as percepções e modos de deslocamento das pessoas, o objetivo geral do presente estudo é analisar percepções do ambiente e a sua relação com a caminhabilidade. A partir da identificação de padrões espaciais e estatísticos da distribuição de categorias de percepção será possível definir principais qualidades espaciais percebidas pelos residentes.

Portanto, faz-se emergente a discussão da caminhabilidade enquanto diretriz de planejamento urbano buscando incentivar interações sociais e o deslocamento ativo (Giles-Corti et al., 2016). Além disso, cidades bem planejadas e mais compactas proporcionam progressos na qualidade de vida da população (Gehl, 2013), sustentabilidade ambiental e vitalidade econômica (Sallis et al., 2016).

\section{Metodologia}

O objetivo principal desta pesquisa é analisar as percepções do ambiente e a sua relação com a caminhabilidade, para tanto, a abordagem de um estudo de caso foi selecionada pela natureza contextual do fenômeno analisado (Yin, 2001). Em função da disponibilidade de dados do Plano de Mobilidade fornecido pelo ITEDES Instituto de Tecnologia, Economia e Desenvolvimento Social e representatividade populacional de uma cidade média brasileira, a cidade de Rolândia-PR foi selecionada. Rolândia é um município localizado na porção norte do estado do Paraná. A cidade tem uma extensão de $459.024 \mathrm{~km}^{2} \mathrm{e}$ uma população estimada em 2017 de 64.726 habitantes (IBGE, 2018) (Figura 2).

Os domicílios selecionados para a pesquisa de OD foram escolhidos a partir do critério de renda, verificado na relação de unidades tributárias da SANEPAR (Companhia de Saneamento do Paraná). Foram aplicados 756 questionários válidos, ou seja $3,76 \%$ de 20.065 domicílios particulares permanentes (IPARDES, 2018). O membro principal da casa foi entrevistado sobre características do domicílio (tipo, número de famílias, número de moradores, tempo de residência, condição de moradia, quantidade de veículos) e informações pessoais, incluindo viagens feitas por cada membro da família no dia anterior. A satisfação quanto ao ambiente do bairro foi quantificada através de perguntas a serem respondidas em uma escala Likert variando de 1 a 5 , relacionadas ao tráfego, deslocamento a pé e qualidade geral do local. 


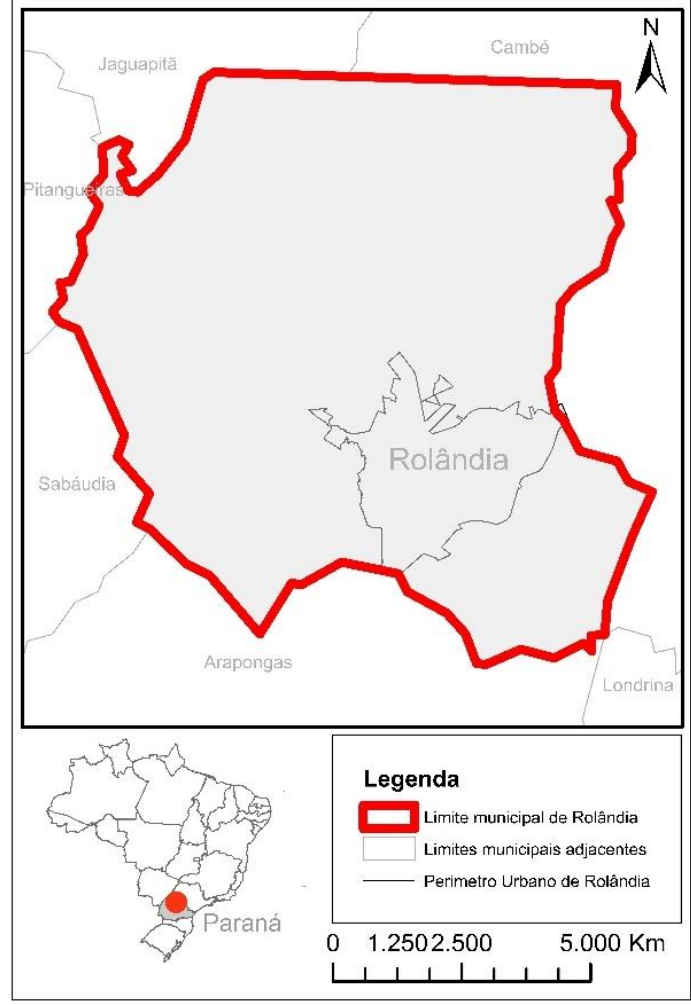

Figura 2. Cidade de Rolândia (fonte: IBGE, 2010, modificado pelas autoras)

Ainda, a percepção em relação ao ambiente construído do bairro foi aferida

subjetivamente através de uma questão aberta indagando sobre observações pertinentes dos respondentes relacionadas à percepções do bairro (ITEDES, 2018). Os respondentes foram geocodificados por meio do Sistema de Informação Geográficas (SIG) no ArcGis 10.5.1 pelo grupo de pesquisa [Grupo de pesquisa] no ano de 2017.

Cada viagem foi composta por dados do pedestre, bicicletas e movimento de tráfego. Um total de 2.731 viagens foram registradas na pesquisa OD de Rolândia, dentre estas 2.097 foram geocodificadas, devido a erros de ortografia, dos nomes das ruas e informações limitadas, apresentados nos demais questionários. As viagens sistematizadas na presente pesquisa consideram apenas respondentes que reportaram a realização de viagens a pé, totalizando em 394 viagens.

A cidade de Rolândia foi georreferenciada pelo grupo de pesquisa [Grupo de pesquisa] permitindo a sistematização das variáveis objetivas e subjetivas da caminhabilidade. Em relação as variáveis objetivas, foram consideradas: densidade residencial, densidade de intersecções, taxa de ocupação de lotes comercias, uso misto do solo ou entropia. Estas variáveis do ambiente construído foram normalizadas por Escore- $Z$ e combinadas no Índice de Caminhabilidade proposto por Frank et al. (2010), que pode ser descrito pela seguinte equação:

\section{Caminhabilidade}

$=[(2 * z$ densidade de intersecções $)$

$+(z$ net densidade residencial)

+ (z taxa de ocupação de usos comerciais)

$+(z *$ diversidade de usos do solo $)]$

A densidade residencial foi definida a partir da proporção entre unidades domiciliares por área do setor censitário, unidade utilizada para todas as análises. Quanto maior o nível dessa variável maior possibilidade da presença de pessoas ativas no bairro. A densidade de intersecções, refere-se à conectividade de ruas, ou seja, maiores possibilidades de escolha de rotas para os pedestres. Esta métrica foi quantificada a partir da razão entre o número de intersecções verdadeiras - entre três ou mais vias - e a área em $\mathrm{m}^{2}$ do setor censitário considerado.Um alto indicador dessa variável corresponde a caminhos mais diretos entre OD. Uso misto do solo está ligado ao grau de diversidade de usos do solo presente em uma unidade de área. Para esta pesquisa, a formula da entropia (Shannon, 1948) foi calculada para a representação do grau de 'mistura' do uso do solo, considerando cinco categorias de uso do solo: residencial, comercial, serviços de entretenimento e institucional. A taxa de ocupação de lotes comerciais, foi medida como a proporção entre a ocupação do edifício comercial pela área do lote comercial. A baixa proporção indica maior área de estacionamentos e baixa proporção menor área de estacionamento, entende-se que esta característica indica a priorização do acesso de pedestres (Jáuregui et al., 2016; Frank et al., 2010).

Os atributos subjetivos, relacionados a percepção do ambiente foram baseados em uma seção especifica dos questionários de OD compostas por observações verbais livres sobre percepções do ambiente do bairro. Somente residentes que reportaram caminhar e verbalizaram percepções de satisfação relacionadas ao ambiente foram geolocalizados e utilizados como dados da pesquisa aqui proposta. Dentre os respondentes $31,21 \%$ do total de 394 viagens, obtiveram percepções, resultando em 120. 
Percepções de bairro foram categorizadas a partir do framework proposto por Pikora et al. (2003) quanto as qualidades ambientais de Funcionalidade, Segurança, Estética e Destinos. Esclarece-se que devido a uma baixa diversidade de percepções relatadas, representá-las através das categorias principais do framewok (categorias) proposto por Pikora et. al. (2013) se mostrou suficiente para retratar a variabilidade dos dados a serem analisados (Tabela 1). É importante enfatizar que alguns residentes verbalizaram mais de uma percepção relacionadas a características diferentes do ambiente. Para dados estatísticos mais consistentes, a escolha metodológica realizada foi considerar somente a categorização da primeira percepção verbalizada como principal, entendendo-se que esta possuiu maior relevância para o respondente. Deve-se apontar também que a grande maioria das percepções em relação à satisfação dos bairros foram apontamentos negativos.

Tabela 1. Categorização das percepções (fonte: elaborada pelas autoras com base em Pikora et al., 2003)

\begin{tabular}{|c|c|c|c|}
\hline $\begin{array}{c}\text { Classificação segundo } \\
\text { Pikora }\end{array}$ & Frequência & $(\%)$ & Percepções predominantes \\
\hline Funcionalidade & 29 & 24.2 & $\begin{array}{l}\text { "Necessidade de quebra-molas nas } \\
\text { ruas" ( } n=18 / \text { trágefo })\end{array}$ \\
\hline Segurança & 17 & 14.2 & $\begin{array}{c}\text { "Precisa de mais segurança no bairro" } \\
(n=15 / \text { segurança pessoal })\end{array}$ \\
\hline Estética & 27 & 22.5 & $\begin{array}{l}\text { "Muitos buracos nas ruas" } \\
\text { ( } n=27 / \text { paisagem urbana) }\end{array}$ \\
\hline Destinos & 47 & 39.2 & $\begin{array}{l}\text { "Estrutura e horários de ônibus ruins" } \\
\text { ( } n=47 / \text { facilidades })\end{array}$ \\
\hline Total & 120 & 100.0 & \\
\hline
\end{tabular}

Para identificar a relação entre variáveis objetivas de caminhabilidade e percepções verbalizadas, mapas foram construídos com o auxílio do software ArcGis 10.5.1 mediante sobreposição das percepções já categorizadas com quintis representativas de níveis de caminhabilidade, a partir da análise de variáveis objetivas. Primeiramente as variáveis objetivas foram quantificadas por setores censitários $(n=70)$ em quintis de baixa, média baixa, média, média alta e alta indicação, divididos de acordo com as quebras naturais dos dados de cada uma das metricas. Foi realizada a sobreposição de dados objetivos e de percepções por setor censitário para análise.

A partir destes dados, o método estatístico de Kruskall-Wallis (Kruskal e Wallis, 1952) foi utilizado para análise da relação entre variáveis objetivas de caminhabilidade e percepções. Este é um teste estatístico não paramétrico que avalia as diferenças entre três ou mais grupos amostrados independentemente em relação a uma única variável que não atende aos pressupostos de normalidade (McKight e Najab, 2010).

Neste estudo as diferenças entre os quatro grupos de percepções categorizadas foram avaliadas em relação aos dados da caminhabilidade objetiva, que não seguem a distribuição normal. Análises estatísticas foram conduzidas com a utilização do software livre para estatística e gráficos R ( R Core Team, 2018). 
Tabela 2. Percepções quantificadas por setor censitário (fonte: elaborada pelas autoras).

\begin{tabular}{|c|c|c|c|c|c|}
\hline $\begin{array}{l}\text { Índice de caminhabilidade } \\
\text { (Total de setores } n=70 \text { ) }\end{array}$ & Baixa $n=11$ & $\begin{array}{l}\text { Média baixa } \\
n=14\end{array}$ & $\begin{array}{c}\text { Média } \\
\mathrm{n}=15\end{array}$ & $\begin{array}{l}\text { Média alta } \\
n=21\end{array}$ & Alta $n=9$ \\
\hline Funcionalidade & $2(8,70 \%)$ & $3(20 \%)$ & $10(24,39 \%)$ & $9(31,03 \%)$ & $5(41,67 \%)$ \\
\hline Segurança & $3(13,04 \%)$ & $1(6,67 \%)$ & $8(19,51 \%)$ & $4(13,80 \%)$ & $1(8,33 \%)$ \\
\hline Estética & $2(8,70 \%)$ & $4(26,67 \%)$ & $9(21,95 \%)$ & $7(24,14 \%)$ & $5(41,67 \%)$ \\
\hline Destinos & $16(69,56 \%)$ & $7(46,66 \%)$ & $14(34,15 \%)$ & $9(31,03 \%)$ & $1(8,33 \%)$ \\
\hline Total & $23(19,16 \%)$ & $15(12,50 \%)$ & $41(34,17 \%)$ & $29(24,17 \%)$ & $12(10 \%)$ \\
\hline $\begin{array}{l}\text { Densidade Residencial } \\
(\text { Total de setores } n=70 \text { ) }\end{array}$ & Baixa $n=11$ & $\begin{array}{l}\text { Média baixa } \\
n=13\end{array}$ & $\begin{array}{c}\text { Média } \\
\mathrm{n}=18\end{array}$ & $\begin{array}{c}\text { Média alta } \\
\mathrm{n}=16\end{array}$ & Alta $n=12$ \\
\hline Funcionalidade & $3(12 \%)$ & $3(25 \%)$ & $8(21,05 \%)$ & $9(34,62 \%)$ & $6(31,58 \%)$ \\
\hline Segurança & $2(8 \%)$ & $2(16,67 \%)$ & $7(18,42 \%)$ & $4(15,38 \%)$ & $2(10,53 \%)$ \\
\hline Estética & $4(16 \%)$ & $2(16,67 \%)$ & $9(23,69 \%)$ & $6(23,08 \%)$ & $6(31,58 \%)$ \\
\hline Destinos & $16(64 \%)$ & $5(41,66 \%)$ & $14(36,84 \%)$ & $7(26,92 \%)$ & $5(26,31 \%)$ \\
\hline Total & $25(20,83 \%)$ & $12(10 \%)$ & $38(31,67 \%)$ & $26(21,67 \%)$ & $19(15,83 \%)$ \\
\hline $\begin{array}{l}\text { Uso misto do } \\
\text { solo/entropia } \\
(\text { Total de setores } n=70 \text { ) }\end{array}$ & Baixa $n=17$ & $\begin{array}{l}\text { Média baixa } \\
n=8\end{array}$ & $\begin{array}{c}\text { Média } \\
\mathrm{n}=16\end{array}$ & $\begin{array}{l}\text { Média alta } \\
\mathrm{n}=28\end{array}$ & Alta $n=1$ \\
\hline Funcionalidade & $5(15,15 \%)$ & $3(15 \%)$ & $7(58,33 \%)$ & $14(25,45 \%)$ & 0 \\
\hline Segurança & $3(9,10 \%)$ & $2(10 \%)$ & $2(16,67 \%)$ & $10(18,18 \%)$ & 0 \\
\hline Estética & $5(15,15 \%)$ & $2(10 \%)$ & $1(8,33 \%)$ & $19(34,55 \%)$ & 0 \\
\hline Destinos & $20(60,60 \%)$ & $13(65 \%)$ & $2(16,67 \%)$ & $12(21,82 \%)$ & 0 \\
\hline Total & $33(27,50 \%)$ & $20(16,67 \%)$ & $12(10 \%)$ & $55(45,83 \%)$ & 0 \\
\hline $\begin{array}{l}\text { Densidade de } \\
\text { cruzamentos } \\
(\text { Total de setores } n=70)\end{array}$ & Baixa $n=18$ & $\begin{array}{l}\text { Média baixa } \\
\mathrm{n}=13\end{array}$ & $\begin{array}{c}\text { Média } \\
\mathrm{n}=15\end{array}$ & $\begin{array}{l}\text { Média alta } \\
\mathrm{n}=18\end{array}$ & Alta $n=6$ \\
\hline Funcionalidade & $4(13,33 \%)$ & $5(20,83 \%)$ & $9(25 \%)$ & $6(27,27 \%)$ & $5(62,5 \%)$ \\
\hline Segurança & $3(10 \%)$ & $4(16,67 \%)$ & $6(16,67 \%)$ & $4(18,18 \%)$ & 0 \\
\hline Estética & $5(16,67 \%)$ & $5(20,83 \%)$ & $8(22,22 \%)$ & $7(31,82 \%)$ & $2(25 \%)$ \\
\hline Destinos & $18(60 \%)$ & $10(41,67 \%)$ & $13(36,11 \%)$ & $5(22,73 \%)$ & $1(12,5 \%)$ \\
\hline Total & $30(25 \%)$ & $24(20 \%)$ & $36(30 \%)$ & $22(18,33 \%)$ & $8(6,67 \%)$ \\
\hline $\begin{array}{l}\text { Taxa de ocupação de lotes } \\
\text { comerciais } \\
(\text { Total de setores } n=70)\end{array}$ & Baixa $n=34$ & $\begin{array}{l}\text { Média baixa } \\
\qquad n=9\end{array}$ & $\begin{array}{c}\text { Média } \\
\mathrm{n}=12\end{array}$ & $\begin{array}{l}\text { Média alta } \\
\mathrm{n}=13\end{array}$ & Alta $n=2$ \\
\hline Funcionalidade & $10(19,61 \%)$ & $7(50 \%)$ & $6(23,08 \%)$ & $5(17,86 \%)$ & $1(100 \%)$ \\
\hline Segurança & $6(11,76 \%)$ & $1(7,14 \%)$ & $2(7,69 \%)$ & $8(28,57 \%)$ & 0 \\
\hline Estética & $10(19,61 \%)$ & $5(35,72 \%)$ & $2(7,69 \%)$ & $10(35,71 \%)$ & 0 \\
\hline Destinos & $25(49,02 \%)$ & $1(7,14 \%)$ & $16(61,54 \%)$ & $5(17,86 \%)$ & 0 \\
\hline Total & $51(42,50 \%)$ & $14(11,67 \%)$ & $26(21,67 \%)$ & $28(23,33 \%)$ & $1(0,83 \%)$ \\
\hline
\end{tabular}

\section{Resultados}

A partir da quantificação objetiva da caminhabilidade e da categorização de percepções relacionadas ao ambiente, realizou-se a sobreposição das informações em mapeamento e a análise estatística. Resultados foram inicialmente obtidos com a 
sistematização de percepções por setor censitário (Tabela 2). As quantidades de percepções categorizadas foram tabuladas em relação ao índice de caminhabilidade e cada uma das variáveis objetivas da forma urbana.

O método estatístico Kruskall-Wallis demonstrou diferenças significantes entre os quatro grupos analisados, indicadas pelo $\mathrm{p}$ valor menor que 0.05 . O teste apresentou maior consistência para percepções relacionadas a funcionalidade, estética e destinos. Entre estas, as percepções de funcionalidade e estética se correlacionam com maiores índices de caminhabilidade (Tabela 3). Percepções de destinos estiveram mais relacionadas à transportes públicos, $\mathrm{e}$ apresentaram coeficiente de variação extremo em relação as demais, indicando uma maior dispersão de dados de caminhabilidade.

Tabela 3. Relação estatística entre escores de caminhabilidade e categorias de percepção (fonte: elaborada pelas autoras).

\begin{tabular}{ccccc}
\hline Kruskall-Wallis & Funcionalidade a & Segurança ab & Estética a & Destinos b \\
\hline Média & 4.23 & 2.91 & 4.19 & 1.40 \\
Mediana & 3.83 & 3.33 & 4.29 & 2.56 \\
Coef. De Variação & 80.62 & 115.99 & 91.97 & 259.99
\end{tabular}

*Kruskal-Wallis chi squared $=11.824$. $p$-value $=0.008011$

Em relação ao Index de caminhabilidade, percepções referentes à destinos foram superiores em locais de baixa, média baixa e média caminhabilidade. Para o setor de média alta caminhabilidade, as percepções predominantes estiveram relacionadas a destinos e funcionalidade. $\mathrm{O}$ setor de alta caminhabilidade obteve maior frequência de percepções sobre funcionalidade e estética (Figura 3).

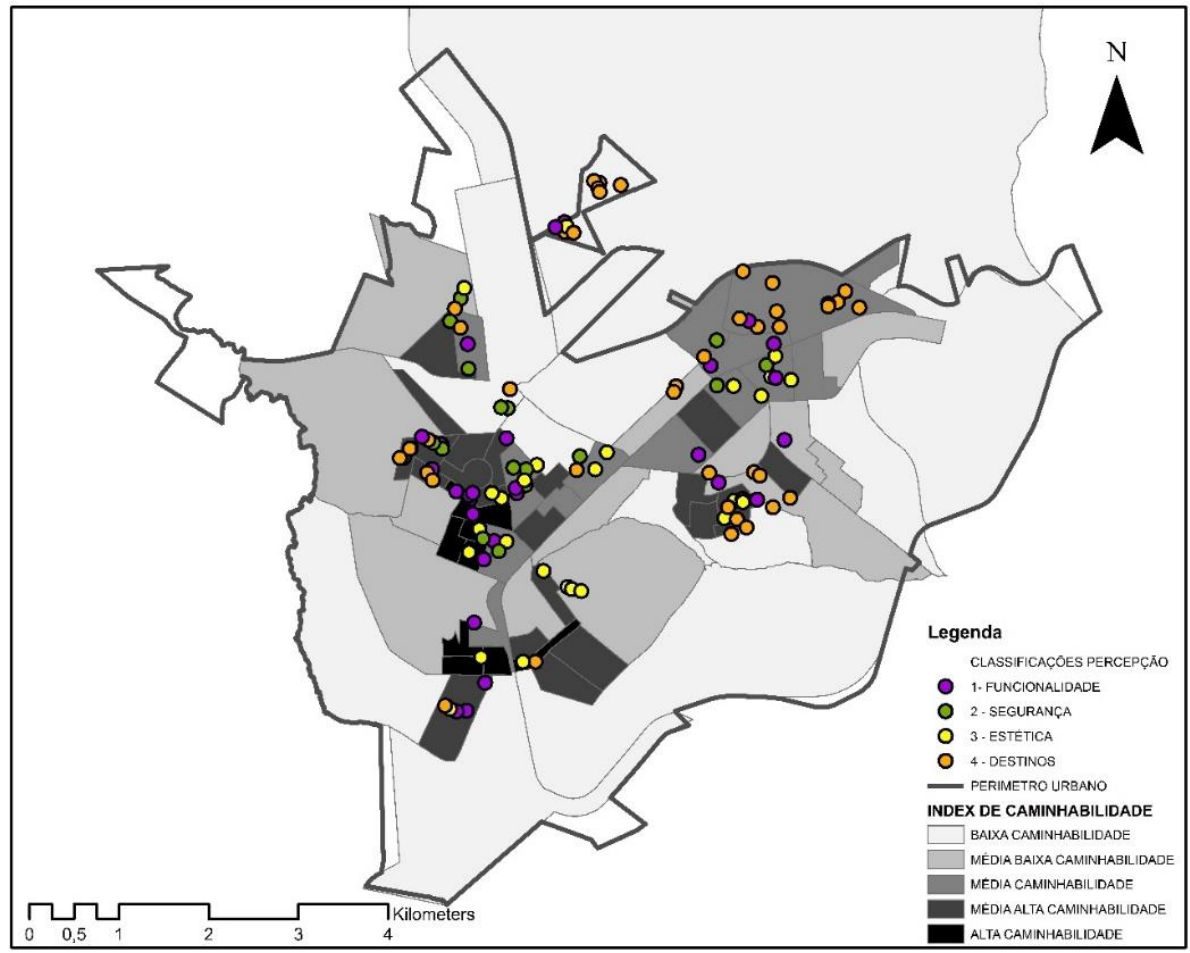

Figura 3. Percepções relacionadas ao Índice de caminhabilidade (fonte: elaborada pelas autoras).

Em relação a variável densidade residencial, percebe-se a ocorrência de maior frequência de percepções relacionadas a destinos em setores censitários de baixa, média baixa e média densidade. Percepções predominates referentes à funcionalidade foram contabilizadas em áreas de média alta densidade e percepções de funcionalidade e estética em alta densidade (Figura 4). 


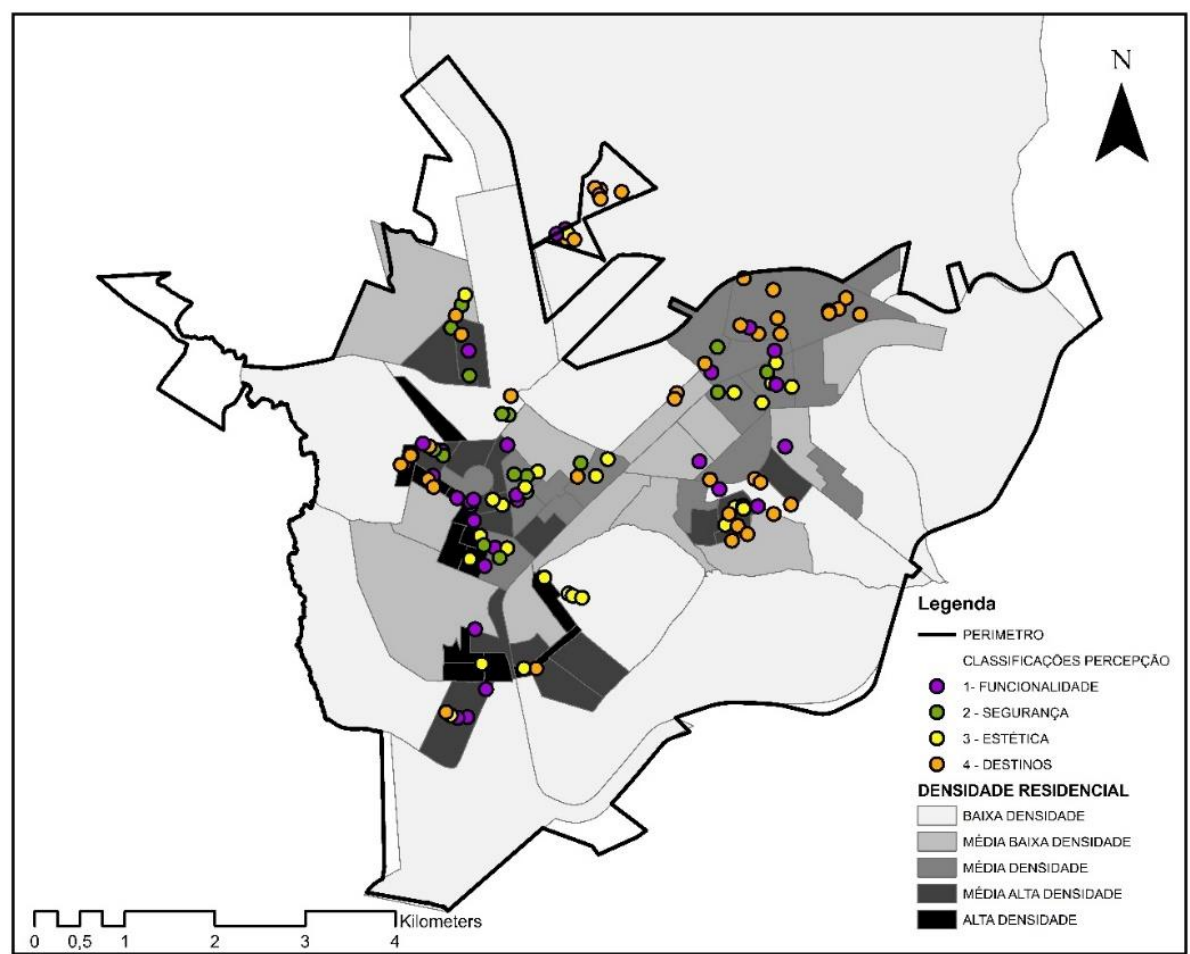

Figura 4. Percepções relacionadas à variável densidade residencial (fonte: elaborada pelas autoras).

Em relação à variável uso misto do solo ou entropia, respondentes não manifestaram suas percepções em setores de alta entropia. Em locais de baixa e média baixa entropia as percepções sobre destinos se mostraram predominantes.
Maior frequência de percepções relacionadas a funcionalidade foram constatadas no setor de média entropia e de estética no setor de média alta entropia (Figura 5).

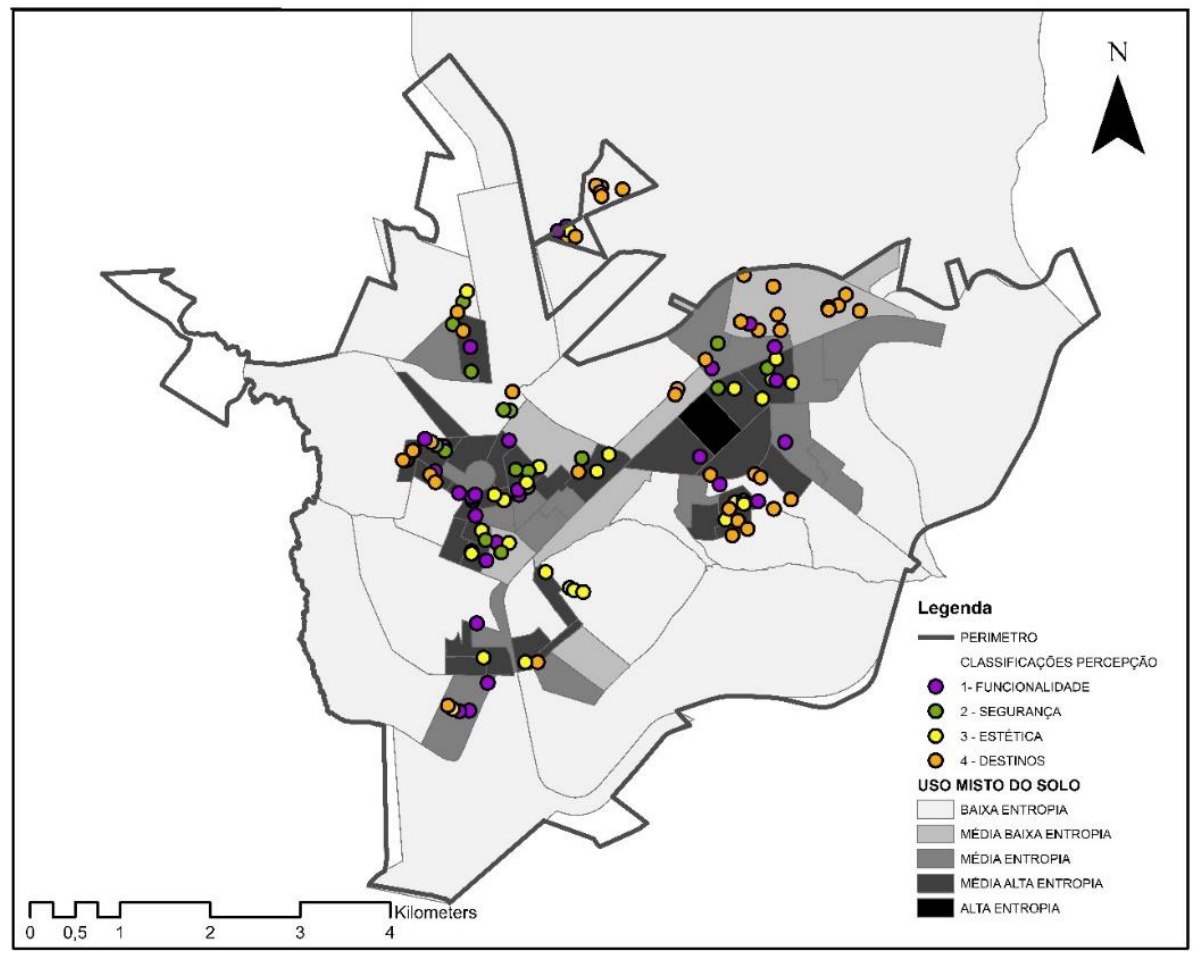

Figura 5. Percepções relacionadas à variável uso misto do solo/entropia (Fonte: elaborada pelas autoras). 
Quanto a variável densidade de cruzamentos maior frequência de percepções relacionadas a destinos ocorreram em setores de baixa, média baixa e média densidade. Quantidades superiores de percepções relacionadas a estética estiveram presentes nos setores de média alta

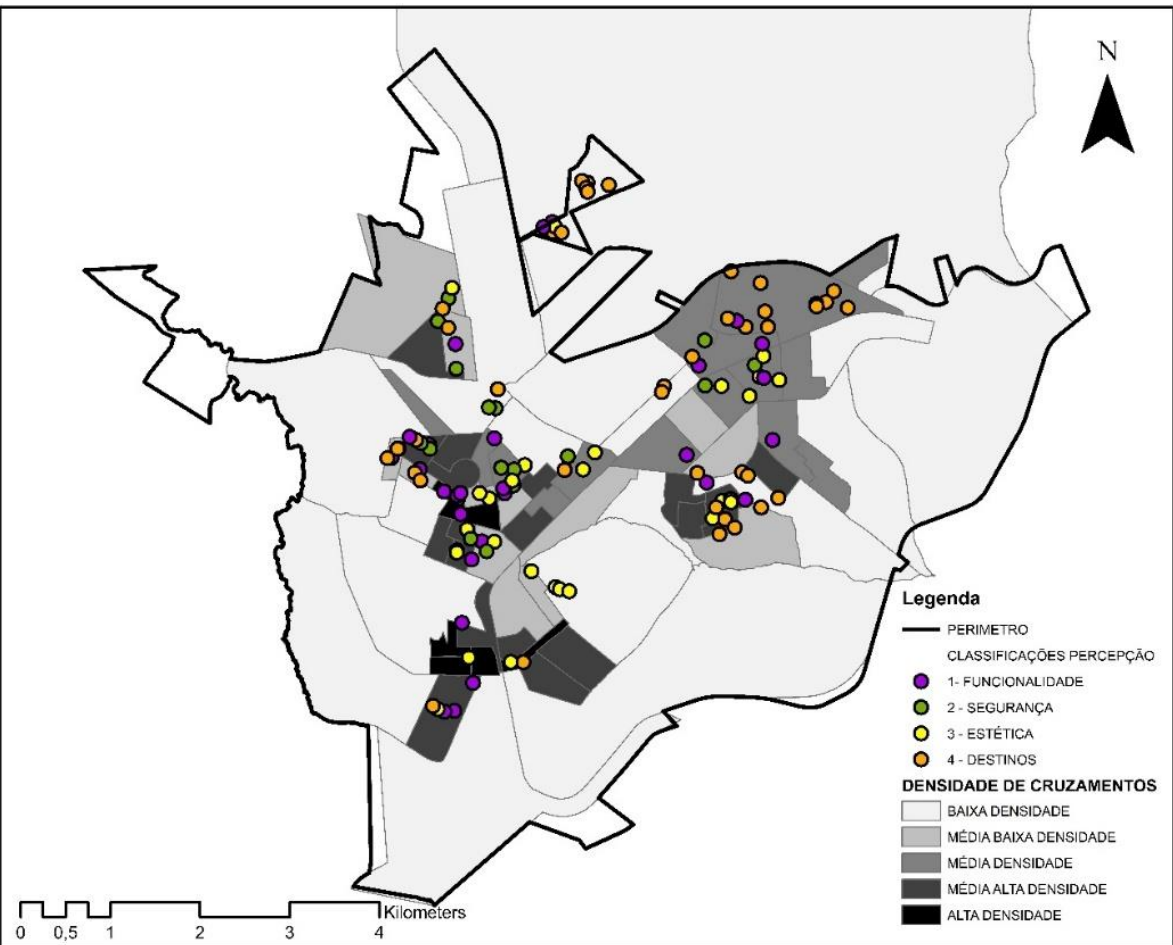

Figura 6. Percepções relacionadas à variável densidade de cruzamentos (Fonte: elaborada pelas autoras).

A variável taxa de ocupação de lotes comerciais compreende maior frequência de percepções relacionadas a destinos em setores de baixa e média taxas. Percepções de funcionalidade se mostraram predominantes no setor de média baixa taxa. densidade. No setor de alta densidade percepções ligadas a funcionalidade se mostraram predominantes (Figura 6). 


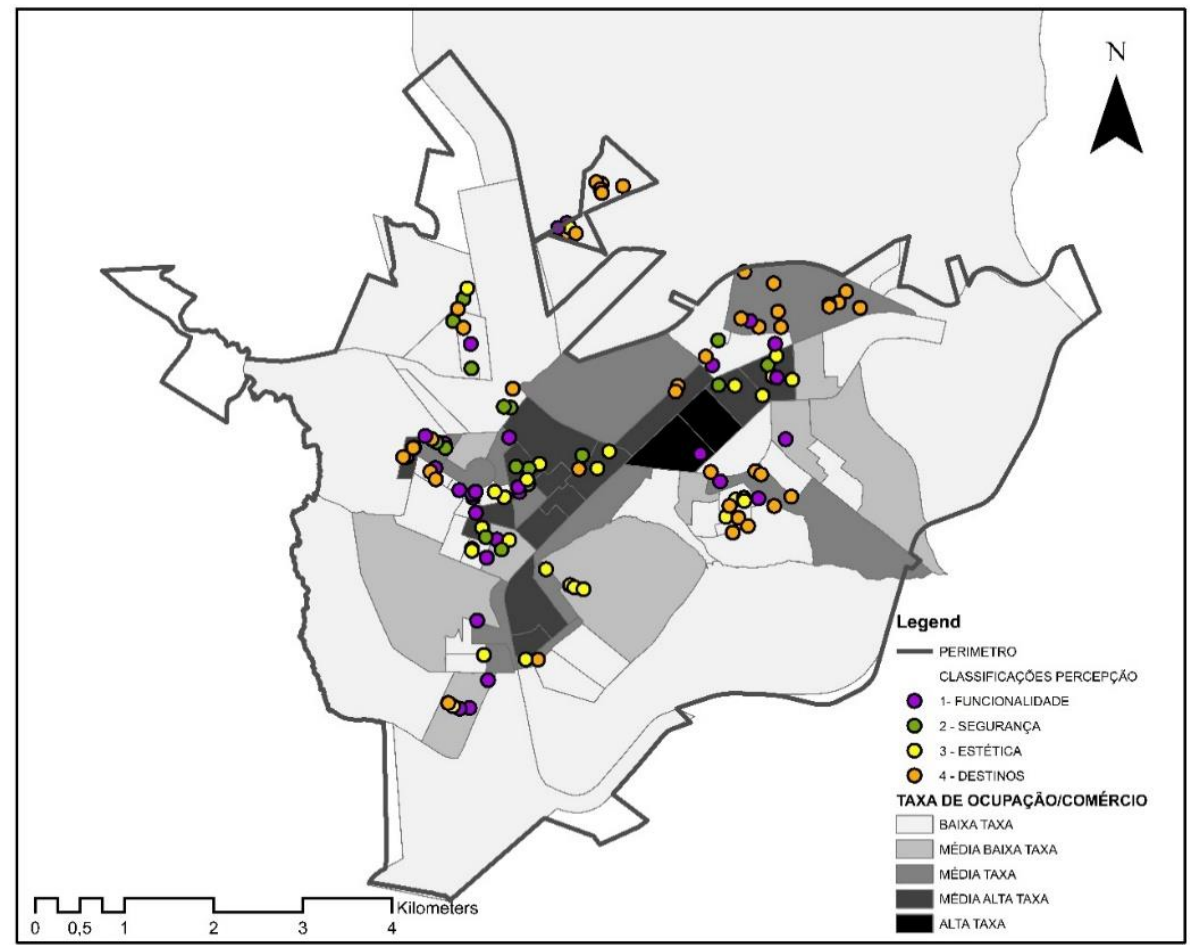

Figura 7. Percepções relacionadas à variável taxa de ocupação de lotes comerciais (onte: elaborada pelas autoras).

\section{Discussões}

O método estatístico não paramétrico de Kruskal-Wallis foi expressivo à presente pesquisa para comprovar a associação entre as variáveis subjetivas e o Index de caminhabilidade. Percepções estiveram relacionadas a funcionalidade e estética demonstrando influência entre o trânsito e o caminho utilizado por pedestres, que devem encontrar formas de atravessar a rua lidando com o perigo do tráfego (Pikora et al., 2003). As percepções de funcionalidade foram mais evidenciadas em verbalizações referentes à falta de dispositivos de controle de trânsito como quebra-molas e a manutenção das calçadas, e percepções de estética a partir de verbalizações relacionadas a presença de buracos nas ruas.

O estudo mostra que os atributos objetivos relacionados ao deslocamento ativo, propostos na teoria de Frank et al. (2010), também foram associados a satisfação de bairro. Em setores com alta caminhabilidade foram verificadas menores quantidades de percepções verbalizadas. Isto poderia ser explicado a partir de características ambientais que influenciam inconscientemente residentes dos bairros (Kamphuis et al., 2010). Esta desconexão indica potencialmente que os residentes não percebem como certas características da vizinhança podem afetar sua atividade física e saúde. Pesquisas anteriores sugerem que adultos com menor renda e escolaridade (Gebel, Bauman e Owen, 2009) e menos atividade física ( Kirtland et al., 2003) são mais prováveis a perceber negativamente características do bairro como tendo menor caminhabilidade do que objetivamente existe. Entende-se que características do design urbano possam ser incorporadas para tornar os bairros de maior caminhabilidade mais atraentes, embora essas características específicas precisem ser identificadas (Lee et al., 2017).

Percepções mostraram-se negativamente associadas a densidade residencial. Infere-se que bairros com baixa densidade residencial, possuem pouca quantidade de pessoas caminhando devido ao baixo número de residências e, portanto, o contato com o ambiente da vizinhança é menor. A partir disto maiores percepções relacionadas a destinos são predominantes, pois o baixo nível de exposição e coesão social geram menores compreensões e familiaridades com características relativas a estética e funcionalidade, presentes na maioria das percepções de residentes de bairros com alta densidade residencial (Jáuregui et al., 2016; Leslie et al., 2005). 
Entende-se que esta relação também pode ser explicada a partir da associação negativa entre destinos percebidos e senso de comunidade, enquanto qualidades estéticas percebidas estão associadas positivamente com o senso de comunidade e maiores níveis de densidade residencial (Grasser, Titze e Stronegger, 2016)

A interconexão entre o uso do solo e o transporte público, percepção predominante na categoria Destinos, resulta em menores distâncias origem destino, incentivando melhores alternativas de mobilidade, como o transporte público, bicicleta e deslocamento a pé (Sallis et al., 2016). Portanto, maiores percepções relacionadas a destinos, em setores de baixa entropia, mostram a desconexão entre a rede viária e a variável uso do solo e a falta de integração entre o planejamento urbano e meio de transporte público.

Melhores percepções sobre conectividade e caminhabilidade estão ligadas ao nível de exposição e compreensão do local (Dewulf et al., 2012). Sendo assim, o baixo nível de exposição dos respondentes sobre o bairro e consequentemente, da menor possibilidade de rotas devido à baixa densidade de cruzamentos implicam em maiores percepções sobre destino.

Associações mais fracas foram encontradas a partir da variável taxa de ocupação de lotes comerciais. Entretanto, entende-se que esta variável da forma urbana proposta por Frank et al. (2010) possui sua validação realizada em cidades Norte americanas onde estão presentes grandes edifícios comerciais marcados por grandes quantidades de estacionamento (Frank et al., 2010). Em vista do porte do estudo de caso analisado aqui, entende-se que esta variável pode não se aplicar a cidade de Rolândia. Porém, mesmo sendo alta a taxa de ocupação de lotes comerciais em determinado setores, as demais variáveis que exercem maior peso na equação da caminhabilidade podem a conferir altos coeficientes.

\section{Conclusões}

Tendo como objetivo da pesquisa a análise das percepções do ambiente e sua relação com a caminhabilidade, padrões de percepção baseado em categorizações propostas por Pikora et al. (2003) relacionados a caminhabilidade objetiva apontada por Frank et al. (2010), foram analisados. A partir de um estudo de caso conduzido na cidade de Rolândia -PR, a consistente associação entre funcionalidade, estética e destinos percebidos com variáveis da caminhabilidade foi observada mediante análise visual de mapas e do teste estatistico de Kruskal - Wallis.

Os resultados desta pesquisa contribuem para diretrizes de projeto urbano sustentáveis, evidenciando características do ambiente que influenciam a percepção das pessoas e incentivam o deslocamento ativo. Estratégias devem ser utilizadas não apenas para melhorar as características do bairro mas também a coesão social, buscando maior envolvimento e percepção dos residentes sobre seu bairro. O estímulo da atividade de pedestres pode proporcionar a melhoria na qualidade de vida e a diminuição de doenças crônicas.

A principal limitação do estudo aqui apresentado foi a utilização de dados secundários obtidos de um estudo OD realizado pela prefeitura de Rolândia considerando questões "abertas" necessárias para a categorização do framework proposto por Pikora et al. (2003), respondidas por 120 pessoas. Os dados coletados não foram levantados na especificidade de analisar a caminhabilidade, mesmo sendo uma fonte importante e coerente de informações. Desta forma, o tamanho da amostra de percepções verbalizadas pelos residentes $(n=120)$ pode ser considerado como uma limitação de pesquisa, possivelmente ocasionando as associações fracas encontradas em relação à caminhabilidade. Porém os resultados obtidos neste estudo poderão ser usados como pré-diretrizes na elaboração e realização de futuros estudos em relação à caminhabilidade.

A falta de atratividade e insegurança percebidas, apresentadas por meio de maiores relações entre o Index de caminhabilidade e percepções de funcionalidade, relacionadas a insegurança com o tráfego, estética e destinos podem ser explicadas a partir do fator socioeconômico (Kamphuis et al., 2010). Embora a presente pesquisa não aborde detalhadamente a questão da renda, é evidente que esse é um fator expressivo em percepções do ambiente. Estudos futuros podem pequisar mais a fundo sua relação tentando desvendar a interferência sobre as percepções de bairro no contexto brasileiro. 


\section{Referências}

Associação Nacional de Transportes Públicos - Antp (2018) Sistema de Informações da Mobilidade Urbana: Relatório geral 2016. ANTP.

Baldock, K.L., Paquet, C., Howard, N.J., Coffee, N.T., et al. (2019) Correlates of Discordance between Perceived and Objective Distances to Local Fruit and Vegetable Retailers. International journal of environmental research and public health. 16 (7). Disponível em: https://doi.org/10.3390/ijerph16071262.

Basolo, V. \& Strong, D. (2002) Understanding the Neighborhood: From Residents' Perceptions and Needs to Action. Housing Policy Debate.13 (1), 83-105. Disponível em:

https://doi.org/10.1080/10511482.2002.9521 436.

Bauman, A.E., Reis, R.S., Sallis, J.F., Wells, J.C., et al. (2012) Correlates of physical activity: Why are some people physically active and others not? The Lancet. 380 (9838), 258-271. Disponível em: https://doi.org/10.1016/S01406736(12)60735-1.

Cervero, R. \& Kockelman, K. (1997) Travel demand and the 3Ds: Density, diversity, and design. Transportation Research Part D: Transport and Environment. [Online] 2 (3), 199-219. Available from: doi:10.1016/S1361-9209(97)00009-6.

Dewulf, B., Neutens, T., Van Dyck, D., de Bourdeaudhuij, I., et al. (2012) Correspondence between objective and perceived walking times to urban destinations: Influence of physical activity, neighbourhood walkability, and sociodemographics. International Journal of Health Geographics. 11 (1), 1. Disponível em: https://doi.org/10.1186/1476-072X-1143.

Frank, L.D., Sallis, J.F., Saelens, B.E., Leary, L., et al. (2010) The development of a walkability index: Application to the neighborhood quality of life study. British Journal of Sports Medicine. 44 (13), 924933. Disponível em:

https://doi.org/10.1136/bjsm.2009.058701.

Gebel, K., Bauman, A. \& Owen, N. (2009) Correlates of non-concordance between perceived and objective measures of walkability. Annals of Behavioral Medicine. 37 (2), 228-238. Disponível em: https://doi.org/10.1007/s12160-009-9098-3.

Gehl, J. (2013) Cidade para pessoas. 2a Edição. São Paulo, Editora Perspectiva.

Giles-Corti, B., Vernez-Moudon, A., Reis, R., Turrell, G., et al. (2016) City planning and population health: a global challenge. The Lancet. 388 (10062), 2912-2924.

Disponível em:

https://doi.org/10.1016/S0140-

6736(16)30066-6.

Grasser, G., Titze, S. \& Stronegger, W.J. (2016) Are residents of high-walkable areas satisfied with their neighbourhood? Journal of Public Health (Germany). 24 (6), 469476. Disponível em: https://doi.org/10.1007/s10389-016-0744-5.

Humpel N, Marshall AL, Leslie E, Bauman A, et al. (2004) Changes in neighborhood walking are related to changes in perceptions of environmental attributes. Annals of Behavioral Medicine. 27 (1), 60-67.

IBGE (2010) Bases e referenciais: Malhas digitais, Censo de 2010. Disponível em: https://mapas.ibge.gov.br/bases-ereferenciais/bases-cartograficas/malhasdigitais

IBGE (2018) Panorama da cidade de Rolândia. 3-4. Disponível em: https://cidades.ibge.gov.br/brasil/pr/rolandia/ panorama.

IPARDES (2018) Caderno Estatístico Município- Município De Rolândia. 1 (1), 143.

ITEDES (2018) PlanMob: Plano Municipal de Mobilidade Urbana de Rolândia-PR.

Jáuregui, A., Salvo, D., Lamadrid-Figueroa, H., Hernández, B., et al. (2016) Perceived and Objective Measures of Neighborhood Environment for Physical Activity Among Mexican Adults, 2011. Preventing Chronic Disease. 13, 1-12. Disponível em: https://doi.org/10.5888/pcd13.160009.

K.Yin, R. (2001) Estudo de caso: Planejamento e Métodos. 2nd edition. São Paulo, Bookman Companhia Editora.

Kamphuis, C.B.M., Mackenbach, J.P., Giskes, K., Huisman, M., et al. (2010) Why do poor people perceive poor neighbourhoods? The role of objective neighbourhood features and psychosocial 
factors. Health and Place. 16 (4), 744-754. Disponível em:

https://doi.org/10.1016/j.healthplace.2010.03. 006.

Kirtland, Porter, D.E., Addy, C.L., Neet, M.J., et al. (2003) Environmental measures of physical activity supports: Perception versus reality. American Journal of Preventive Medicine. 24 (4), 323-331.

Disponível em: https://doi.org/10.1016/S07493797(03)00021-7.

Kruskal, W.H. \& Wallis, W.A. (1952) Use of Ranks in One-Criterion Variance Analysis. Journal of the American Statistical Association. 47 (260), 583-621.

Lee, S.M., Conway, T.L., Frank, L.D., Saelens, B.E., et al. (2017) The Relation of Perceived and Objective Environment Attributes to Neighborhood Satisfaction. Environment and Behavior. 49 (2), 136-160. Disponível em: https://doi.org/10.1177/0013916515623823.

Leslie, E. \& Cerin, E. (2008) Are perceptions of the local environment related to neighbourhood satisfaction and mental health in adults? Preventive Medicine. 47 (3), 273278. Disponível em: https://doi.org/10.1016/j.ypmed.2008.01.014.

Leslie, E., Saelens, B., Frank, L., Owen, N., et al. (2005) Residents' perceptions of walkability attributes in objectively different neighbourhoods: A pilot study. Health and Place.11 (3), 227-236. Disponível em: https://doi.org/10.1016/j.healthplace.2004.05. 005 .

Mackenbach, J.D., Lakerveld, J., van Lenthe, F.J., Bárdos, H., et al. (2016) Exploring why residents of socioeconomically deprived neighbourhoods have less favourable perceptions of their neighbourhood environment than residents of wealthy neighbourhoods. Obesity Reviews. 17 (May), 42-52. Disponível em:

https://doi.org/10.1111/obr.12375.

McKight, P.E. \& Najab, J. (2010) KruskalWallis Test. The Corsini Encyclopedia of Psychology. (1).

Pikora, T., Giles-Corti, B., Bull, F., Jamrozik, K., et al. (2003) Developing a framework for assessment of the environmental determinants of walking and cycling. Social Science and Medicine. 56 (8), 1693-1703.

R Core Team (2018) R: A Language and Environment for Statistical Computing. 2018. R Foundation for Statistical Computing.

Sallis, J.F., Bull, F., Burdett, R., Frank, L.D., et al. (2016) Use of science to guide city planning policy and practice: how to achieve healthy and sustainable future cities. The Lancet. 388 (10062), 2936-2947. Disponível em: https://doi.org/10.1016/S01406736(16)30068-X.

Shamsuddin, S., Abu Hassan, N.R. \& Ilani Bilyamin, S.F. (2018) Walkable in Order to be Liveable. Journal of ASIAN Behavioural Studies. 3 (7), 165. Disponível em: https://doi.org/10.21834/jabs.v3i7.269.

Shannon, C.E. (1948) A Mathematical Theory of Communication. Bell System Technical Journal. 27 (3), 379-423.

Disponível em: https://doi.org/10.1002/j.15387305.1948.tb01338.x.

Southworth, M. (2005) Designing the Walkable City. Journal of Urban Planning and Development. 131 (4), 246-257. Disponível em: https://doi.org/10.1061/(asce)07339488(2005)131:4(246). 


\section{Tradução do título, resumo e palavras-chave}

Perceptions of the built environment and their association with objective walkability

Abstract. With the increasing burden of motorized transport, urban qualities have guided researchers to understand the influence of urban form on active travel. One of the strategies to evaluate the built environment is walkability, understood as the extent to which urban form may or may not be conducive to walking. This quality may be analyzed subjectively or objectively, considering perceptions or quantifications of environmental characteristics. Therefore, the general research objective is to analyze perceptions of the built environment related to walkability in a brazilian city. Perceptions were extracted from the Origin-Destination survey carried out for an Urban Mobility Plan of a case study - Rolândia/PR An Index was systematized as an objective and composed walkability measure, by census tracts, and analyzed in map overlays in relation to perceptions through the Kruskal-Wallis statistical procedure. Results indicate that functional, aesthetic and destination perceptions were associated to walkability, while perceptions of safety were less relevant. The study contributes for mobility guidelines in incorporating environmental perceptions as support to active travel to promote the sustainable city.

Keywords. built environment; walkability; sustainability; perception.

Editor responsável pela submissão: Renato Saboya.

Licenciado sob uma licença Creative Commons. 